\title{
Transverse Energy Measurement in Au+Au Collisions by the STAR Experiment
}

\author{
Raghunath Sahoo (for the STAR Collaboration)* \\ Institute of Physics, Bhubaneswar, India - 751005 and \\ SUBATECH, 4, Rue Alfred Kastler, BP 20722 - 44307 Nantes Cedex 3, France
}

\begin{abstract}
Transverse energy $\left(E_{T}\right)$ has been measured with both of its components, namely hadronic $\left(E_{T}^{\text {had }}\right)$ and electromagnetic $\left(E_{T}^{e m}\right)$ in a common phase space at mid-rapidity for $62.4 \mathrm{GeV}$ $\mathrm{Au}+\mathrm{Au}$ collisions by the STAR experiment. $E_{T}$ production with centrality and $\sqrt{s_{N N}}$ is studied with similar measurements from SPS to RHIC and is compared with a final state gluon saturation model (EKRT). The most striking feature is the observation of a nearly constant value of $E_{T} / N_{c h} \sim 0.8 \mathrm{GeV}$ from AGS, SPS to RHIC. The initial energy density estimated by the boost-invariant Bjorken hydrodynamic model, is well above the critical density for a deconfined matter of quarks and gluons predicted by lattice QCD calculations.
\end{abstract}

\section{INTRODUCTION}

Extreme conditions of high temperature and densities could be created in relativistic heavy ion collisions making a scenario to study the deconfined state of quarks and gluons called Quark Gluon Plasma (QGP). The interactions can be characterized in terms of the global variables like transvesre energy and the charged particle multiplicity. These variables are closely related to the collisions geometry and are very important in understanding the global properties of the system created during heavy ion collisions. $E_{T}$ is the energy created transverse to the beam direction and is generated by the initial scattering of the partonic constituents of the incoming nuclei and probably also by the produced partons and hadrons [1, 2]. $E_{T}$ measurement gives an estimation of the initial Bjorken energy density $\left(\epsilon_{B j}\right)$ produced in the fireball and also helps in studying the particle production mechanism.

\section{DATA ANALYSIS}

We have analyzed the $62.4 \mathrm{GeV} \mathrm{Au+Au} \mathrm{minimum-bias} \mathrm{STAR} \mathrm{data} \mathrm{for} \mathrm{RHIC} \mathrm{run} \mathrm{2004.} \mathrm{The}$ detectors used in this analysis include the Time Projection Chamber (TPC) and Barrel Electromagnetic Calorimeter (BEMC) in a common phase space $(0<\eta<1$ and full azimuthal coverage). TPC uncorrected mid-rapidity multiplicity within $|\eta|<0.5$ and $\left|V_{z}\right|<30 \mathrm{~cm}$, is used for the centrality selection. The analysis method adopted here provides an independent event-by-event measurement of both the components of transverse energy i.e. $E_{T}^{h a d}$ and $E_{T}^{e m}$. $E_{T}^{h a d}$ is obtained from the TPC reconstructed tracks after taking into account the long-lived neutral hadrons which could not be detected by the TPC. $E_{T}^{e m}$ is estimated from the energy deposited in the calorimeter towers after correcting for the hadronic contaminations, by projecting hadronic tracks onto BEMC. The details of the transverse energy estimation procedure is discussed in Ref. [4, 5].

*Electronic address: Raghunath.Sahoo@subatech.in2p3.fr 

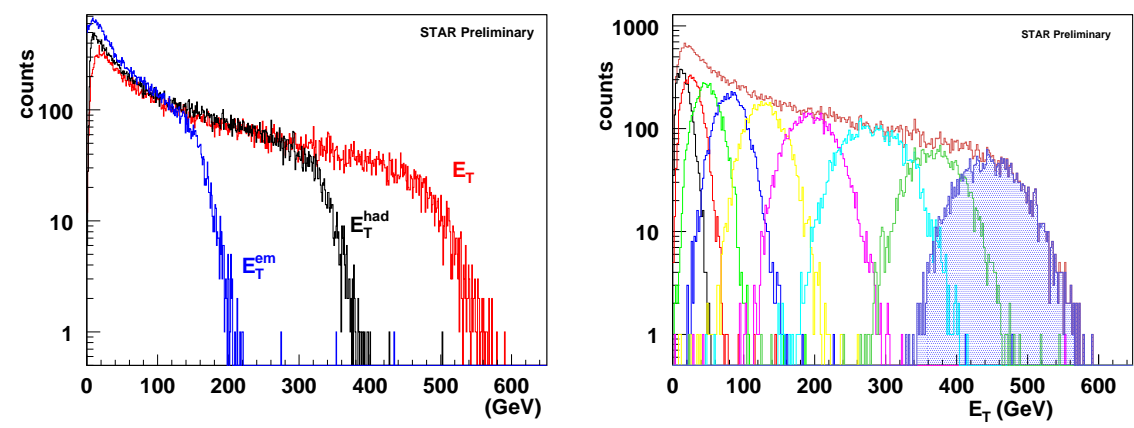

FIG. 1: The minimum-bias distributions of $E_{T}$ and its components (left) and $E_{T}$ distribution for all centrality classes (right), for $62.4 \mathrm{GeV} \mathrm{Au+Au} \mathrm{collisions.}$
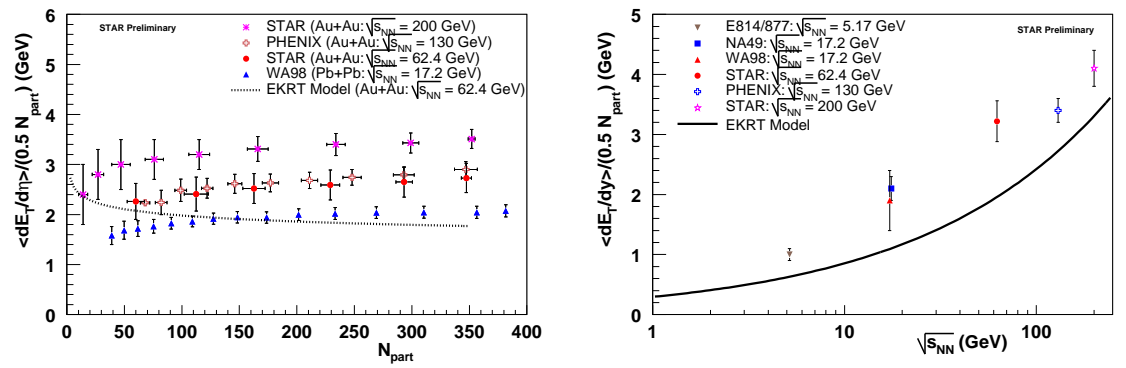

FIG. 2: The mid-rapidity $\left\langle d E_{T} / d \eta>\right.$ per $N_{\text {part }}$ pair vs $N_{\text {part }}$ (left) compared to the EKRT model prediction for $62.4 \mathrm{GeV} \mathrm{Au}+\mathrm{Au}$ data and $\left\langle d E_{T} / d y>\right.$ per $N_{\text {part }}$ pair vs $\sqrt{s_{N N}}$ showing the logarithmic growth from AGS-SPS to RHIC (right). The solid line is the EKRT model prection.

\section{RESULTS AND DISCUSSION}

Figure 1 (left) shows the event-by-event minimum-bias $E_{T}$ and its components with the right figure showing $E_{T}$ distribution for all centrality classes which are gaussian in nature. The minimumbias distributions show a peak and a sharp drop-off at low $E_{T}$ end corresponding to peripheral collisions. It reaches a broad plateau at the middle which corresponds to mid-central collisions. This is dominated by nuclear geometry. The higher values of $E_{T}$ correspond to the most central collisions having a "knee" leading to a fall off which is very steep for large acceptances and less for small acceptances. For the top $5 \%$ central collisions $\left\langle d E_{T} / d \eta\right\rangle=474 \pm 51 \mathrm{GeV}$. The variation of $\left\langle d E_{T} / d \eta\right\rangle$ per $N_{\text {part }}$ pair as a function of centrality is shown in Figure 2 (left) for $62.4 \mathrm{GeV}$ $\mathrm{Au}+\mathrm{Au}$ collisions along with similar measurements from $\mathrm{Pb}+\mathrm{Pb} 17.3 \mathrm{GeV}$ at SPS 6] and $\mathrm{Au}+\mathrm{Au}$ 130 [7], $200 \mathrm{GeV}$ [4] at RHIC. The corresponding EKRT model [3] prediction for $62.4 \mathrm{GeV} \mathrm{Au}+\mathrm{Au}$ collisions is shown by the dotted line. The data show a similar centrality behavior at different energies, whereas the EKRT model doesn't agree with the data. For top central collisions, the variation of $\left\langle d E_{T} / d y>\right.$ per $N_{\text {part }}$ pair as a function of $\sqrt{s_{N N}}$ is shown in Figure 2 (right) with similar measurements at other energies along with the EKRT model prediction which is shown by the thick line. $\left\langle d E_{T} / d y>/\left(0.5 N_{\text {part }}\right)\right.$ increases logarithmically with $\sqrt{s_{N N}}$. However, the EKRT model shows an underestimation of data at various energies.

In order to understand the systematic growth of transverse energy with $\sqrt{s_{N N}}$, the centrality 

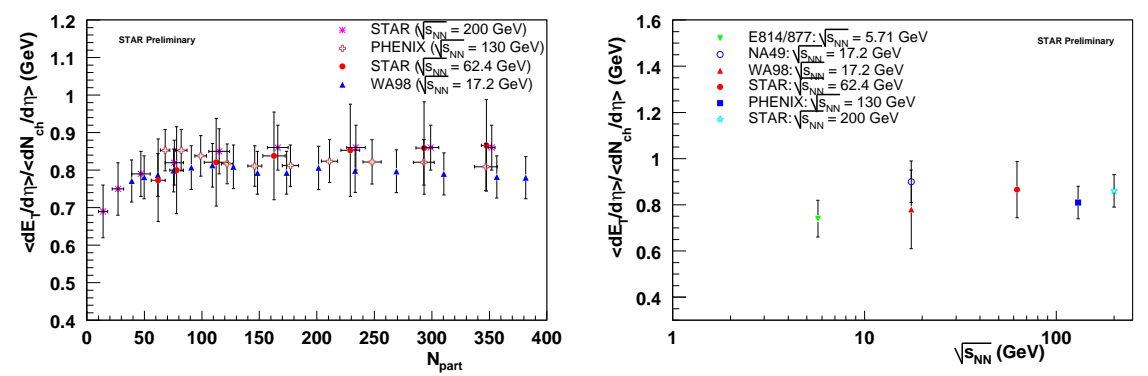

FIG. 3: $\left\langle d E_{T} / d \eta>/<d N_{c h} / d \eta>\right.$ vs $N_{\text {part }}$ (left) and $\left\langle d E_{T} / d \eta>/<d N_{c h} / d \eta>\right.$ vs $\sqrt{s_{N N}}$.

dependence of $\left\langle d E_{T} / d \eta\right\rangle /\left\langle d N_{c h} / d \eta\right\rangle \equiv E_{T} / N_{c h}$ is shown in Figure 3 (left). $E_{T} / N_{c h}$ shows an centrality independent value of $\sim 0.8 \mathrm{GeV}$ except for peripheral collisions which are affected by hydrodynamic flow. The assumption of an isentropic expansion of the fireball explains the the decrease in $E_{T} / N_{c h}$ for peripheral collisions. A similar centrality behavior of $\left\langle p_{T}>[4]\right.$ suggests that energy goes for new particle production, instead of increasing the mean energy of the particle. Taking top central events, the value of $E_{T} / N_{c h}$ for different energies is shown in Figure 3 (right) as a function of $\sqrt{s_{N N}}$ from SPS to RHIC. This ratio is an important barometric measure of internal pressure in ultra-dense matter produced in heavy ion collisions [8]. This centrality and energy independence of $E_{T} / N_{c h}$ has been explained by statistical hadron gas model and is found to be associated with the freeze-out of the fireball [9, 10]. The most important information from this observation is that, irrespective of the initial conditions which are controlled by the $\sqrt{s_{N N}}$ and centrality, the system evolves to the same final state at freeze-out. The observed saturated value of $E_{T} / N_{c h}$ could be taken as its value in the pre-hadronic state, as $T_{c h} \sim T_{c}$, the critical temeprature for the quark-hadron phase transition.

Next we have studied the Bjorken energy density [11] produced in the top central $\mathrm{Au}+\mathrm{Au}$ collisions, which is shown in Figure 4 (left) along with similar data at other RHIC energies. The value of the energy density produced in top central $\mathrm{Au}+\mathrm{Au}$ collisions at $62.4 \mathrm{GeV}$ is found to be $3.65 \pm 0.39 \mathrm{GeV} / \mathrm{fm}^{3}$ (taking formation time, $\tau=1 \mathrm{fm} / \mathrm{c}$ ). This is well above the lattice QCD predictions 12] for a decofined state of quarks and gluons. This figure shows an logarithmic increase of $\epsilon_{B j} . \tau$ and its extrapolation to LHC energy gives a value of $9.42 \pm 0.55 \mathrm{GeV} \mathrm{fm}^{-2} c^{-1}$, based on the assumption that Bjoken model holds good at higher energies. We have shown the centrality dependence of $\epsilon_{B j} . \tau$ in Figure 4 (right), where one observes that higher energy densities are produced in more central collisions. Further more we have also studied the excitation functions of $\left\langle d E_{T} / d \eta\right\rangle /\left(0.5 N_{\text {part }}\right)$ and $\left\langle d N_{c h} / d \eta>/\left(0.5 N_{\text {part }}\right)\right.$ which show a logarithmic behavior. The details of which could be found elsewhere [5].

The electromagnetic fraction of the total transverse energy $\left.\left.\left(<d E_{T}^{e m} / d \eta\right\rangle /<d E_{T} / d \eta\right\rangle\right)$ for top central $\mathrm{Au}+\mathrm{Au}$ collisions for $62.4 \mathrm{GeV}$, is studied as a function of $\sqrt{s_{N N}}$, along with similar data from AGS-SPS to RHIC for different colliding species. This is shown in Figure 5 (left). The value of this ratio at $62.4 \mathrm{GeV}$ is $0.32 \pm 0.03$ and the ratio shows a very slow increase with energy. This increase is consistent with the meson dominance of the matter at higher energies. Figure [5] (right) shows that this ratio is independent of the collision centrality.

\section{SUMMARY}

The mid-rapidity measurement of $E_{T}$ for $62.4 \mathrm{GeV} \mathrm{Au+Au}$ collisions is presented. The centrality and center of mass energy behavior of $E_{T}$ production is studied and compared with 

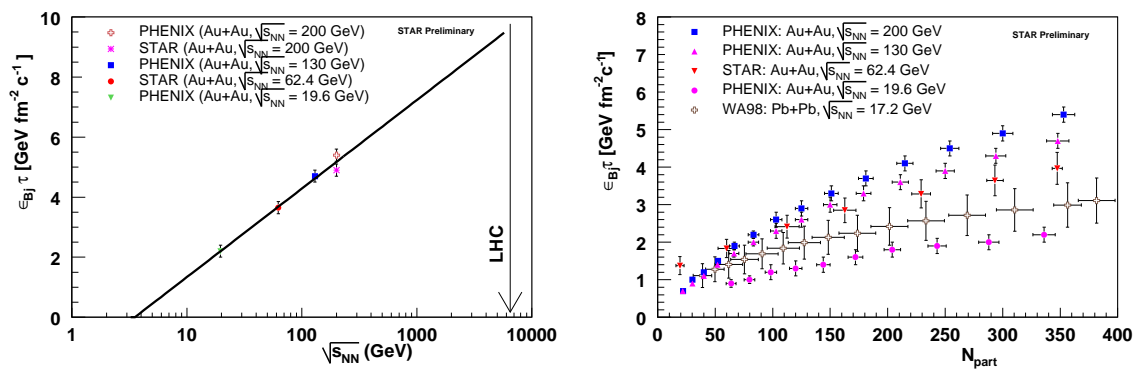

FIG. 4: $\left\langle d E_{T}^{e m} / d \eta\right\rangle /\left\langle d E_{T} / d \eta\right\rangle$ vs $\sqrt{s_{N N}}$ for a number of systems from SPS to RHIC (left) and $<d E_{T}^{e m} / d \eta>/<d E_{T} / d \eta>$ vs $N_{\text {part }}$.
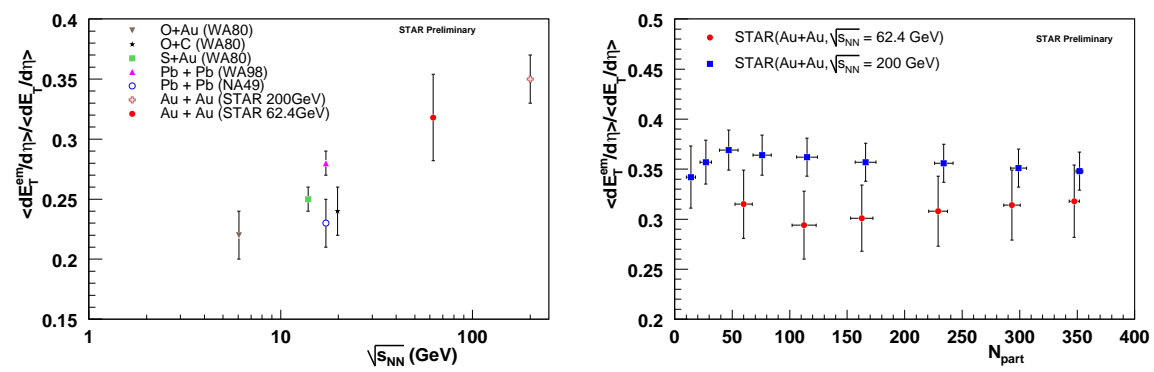

FIG. 5: $\left\langle d E_{T}^{e m} / d \eta>/<d E_{T} / d \eta>\right.$ vs $\sqrt{s_{N N}}$ for a number of systems from SPS to RHIC (left) and $<d E_{T}^{e m} / d \eta>/<d E_{T} / d \eta>$ vs $N_{\text {part }}$.

similar data at other energies and with the EKRT gluon saturation model. The observation of a centrality and $\sqrt{s_{N N}}$ independent, nearly constant value of $E_{T} / N_{c h} \sim 0.8 \mathrm{GeV}$ from AGS, SPS to RHIC has been understood to be associated with freeze-out of the fireball. The initial energy density estimated by the boost-invariant Bjorken hydrodynamic model, is well above the lattice QCD value for a deconfined matter of quarks and gluons. Taking similar colliding species i.e. $\mathrm{Au}+\mathrm{Au}$, the $\epsilon_{B j} . \tau$ has been predicted for LHC, based on the measurements at RHIC.

\section{References}

[1] M. Jacob and P.V. Landshoff, Mod. Phys. Lett. A1, 657 (1986).

[2] X.N. Wang, Phys. Rep. 280, 287 (1997).

[3] K.J. Eskola, K. Kajantie, P.V. Ruuskanen and K. Tuominen, Nucl. Phys. B 570, 379 (2000).

[4] J. Adams et al., STAR Collaboration, Phys. Rev. C 70, 054907 (2004).

[5] Raghunath Sahoo, Ph.D. Thesis, 2007, STAR Collaboration, Preprint: 0804.1800 [nucl-ex].

[6] M.M. Aggarwal et al., WA98 Collaboration, Eur. Phys. J. C 18, 651 (2001).

[7] K. Adcox et al., PHENIX Collaboration, Phys. Rev. Lett. 87, 052301 (2001).

[8] M. Gyulassy, Lecture Notes in Physics, 583, 37 (2001).

[9] J. Cleymans, R. Sahoo, D.P. Mahapatra, D.K. Srivastava and S. Wheaton, Phys. Lett. B 660, 172 (2008) and Preprint: 0803.3940 [hep-ph], to appear in J. Phys. G: Nucl. Part. Phy..

[10] J. Cleymans et al., Eur. Phys. J: Spec. Topics 155, 13 (2008).

[11] J.D. Bjorken, Phys. Rev. D 27, 140 (1983).

[12] F. Karsch, Nucl. Phys. A 698, 199c (2002). 